\section{A Fiberoptic-based System for Integrating Photosynthetically Active Radiation in Plant Canopies}

\author{
William L. Bauerle ${ }^{1}$ and Joseph D. Bowden \\ Department of Horticulture, Clemson University, Clemson, SC 29634-0319
}

Additional index words. Acer rubrum, light interception, leaf area index

\begin{abstract}
This report describes a system for integrating photosynthetically active radiation (PAR) using fiberoptics. Many photoelectric sensors or 1-m-long line sensors that integrate individual interception points for spatial averaging were replaced with fiberoptics, which integrate interception points. Depending on the positioning of optical fibers and the amount of fibers terminated at a PAR sensor, whole-plant, canopy layer, and individual leaf light interception can be determined. The use of fiberoptics has the added advantage of being very small in comparison to the bulk of a typical quantum sensor. The fiberoptic-based system potentially is a more accurate, less expensive method to integrate PAR throughout plant canopies than PAR sensors.
\end{abstract}

Characterization of the light environment in a spatially and temporally variable plant canopy or orchard requires adequate description of the repeating unit of the planting pattern (Jackson, 1980). A single point photosynthetically active radiation (PAR) sensor measures the flux density of quanta within a selected spectral waveband, within the PAR wavelength range (400 to $700 \mathrm{~nm}$ ) (Pearcy, 1989). However, this method is limited because it only quantifies light at a single point. As is often the case when using single point PAR sensors, inadequate spatial sampling procedures occur (Kyle et al., 1977; Baldocchi and Collineau, 1994; Salimen et al., 1983). Alternatively, integrated light measurements can be obtained from line quantum sensors, which are often used in crops that are row oriented (Pearcy, 1989). Trying to collect information about a spherical object with light bars necessitates the use of multiple linear light bars positioned orthogonally, which can be arduous. Regardless of technique, measurements of the radiation transfer through canopies strive to adequately characterize the foliage interception in canopies.

The most economic photoelectric sensors with spectral responses in the PAR region, silicon cells (Si) (Pearcy, 1989) and gallium arsenide phosphide (GaAsP) (Pearcy, 1989) sensors, do not integrate PAR over many points and require a large amount of data channels and logger memory. In addition, such PAR data are subject to question due to the shiny surface of photoelectric sensors, a potential cause of poor responses at low angles of incidence (Pearcy, 1989). When mounted directly on several leaves and at different canopy layers, GaAsP photocells are capable of sampling various points within a canopy (Gutschick et al. 1985). However, fiberoptics offer an advantage since they allow integration of 2 to $>100$ light

Received for publication 1 Apr. 2003. Accepted for publication 9 Sept. 2003. Technical contribution of the South CarolinaAgriculture and Forestry Research System, Clemson University.

${ }^{1}$ Corresponding author; e-mail bauerle@clemson.edu. interception points into one PAR and or solar radiation sensor reading.

Immature or widely spaced crop canopies such as woody nursery vegetation and orchards vary in either two or three dimensions. Models that characterize the light environment within such canopies are complicated and validation of the probability of beam penetration through foliage, such as the Norman and Wells (1983) three-dimensional radiation transfer model, justify the need for an inexpensive and integrated canopy light interception with an inexpensive fiberoptic-based system overcomes the common limitations mentioned above, especially when the focus is to measure different canopy layers and validate a multilayer model. Integration of the quantity of light over numerous points in as large an area as necessary is not restricted by geometric shapes and therefore, drastically reduces the number of PAR sensors required. et al., 2004) on intracanopy interactions of PAR in Acer rubrum L. (red maple). In this report, we test a fiberoptic-based PAR integration system and examine three canopy layers, in three independent trees, and in comparison to paired line quantum sensor values in red maple canopies. Validation was achieved by comparing measurements of fiberoptic PAR to an orthogonal grid of PAR line sensors.

\section{Materials and Methods}

The study was conducted at the Clemson University Calhoun Field Laboratory site in Clemson, S.C., during 2002. A full descripBauerle et al. (2002). Briefly, containerized red maple saplings were spaced $1.25 \mathrm{~m}$ center to center. Three-year-old stock in 57-L Spin Out (Nursery Supplies Inc.) treated plastic pots contained a mixture of pine bark and sand $(20: 1, \mathrm{v} / \mathrm{v})$, fertilized with $8.3 \mathrm{~kg} \cdot \mathrm{m}^{-3}$ of Nutricote 20N-3.0P-8.3K type 360 (ChisoAsahi Inc.). PAR measurement technique. Measurement of

This report is part of a larger study (Bauerle tion of the site and plant material is given in
Paired line and fiberoptic PAR measurements. On 22 July 2002 three uniform red maple trees in the central portion of the plot were randomly chosen for intensive measurement of PAR interception. Each tree was placed within a telescopic poly vinyl chloride (PVC) rack (Fig. 1) measuring $1 \mathrm{~m}$ wide and $1 \mathrm{~m}$ long for $9 \mathrm{~d}$. PVC pipe $5 \mathrm{~cm}$ in diameter cut into 1 -m lengths was used for the bottom four legs and then $3.75-\mathrm{cm}$ and $2.5-\mathrm{cm}$-diameter PVC was slid inside of the 5-cm tubes, respectively. The four legs had peg holes drilled every 10 $\mathrm{cm}$ on three sections. When fully extended the rack was $3 \mathrm{~m}$ high. The rack supported fourline quantum sensors (LI-COR Inc., Lincoln, Neb.) placed orthogonal in a square grid. Each line sensor was placed $40 \mathrm{~cm}$ perpendicular to the tree stem in an north, south, east, and west orientation. The canopies were each divided into three layers (I, II, and III) by splitting the length of the live crown into equal thirds. The telescopic rack was moved to the median of each layer for $3 \mathrm{~d}$ per layer and after the three layers were sampled, the rack was moved to the next sample tree. In all, three trees were sampled at three layers for $3 \mathrm{~d}$ per canopy layer. The line sensors sampled PAR every $1 \mathrm{~min}$ and then recorded a 15-min average (CR10x; Campbell Scientific, Logan, Utah).

The fiberoptic technique to integrate PAR used multimode fiberoptic bundles that sampled canopy light interception incident at three layers of the canopy boundary. The multimode optical fiber was specifically chosen not to interfere with wavelength transmission (Clifford R. Pollock, Cornell University, personal communication). To manufacture the optical sensors, we followed Secore Recommended Procedure SRP-005-005 and SRP-005-006 (Corning Cable Systems, 2001). The specific technical details are available from the author.

To sample PAR interception via fiberoptics, each of the three intensive light sample trees were fit with three Corning optical cables (one per canopy layer) (Fig. 1). The four sets of twelve microfibers per cable were placed at the boundary edge of the median of each of the three canopy layers per tree at a $45^{\circ}$ angle facing each cardinal direction (north, south, east, and west), fastened to and supported by a 7-mm wooden dowel, and secured to the main stem with plastic fasteners. This integrated 48 $62.5-\mu \mathrm{m}$ irradiance interception points per canopy layer. All sensors were sampled every $1 \mathrm{~min}$ at a $5000-\mu \mathrm{V}$ sensitivity and 15 -min averages were logged (CR7x, Campbell Scientific, Logan Utah). At any one time, three trees and three canopy layers were continuously sampled for PAR interception by the fiberoptic-based system, whereas the orthogonal grid of line quantum sensors could sample only one layer on one tree.

PAR sensor scaling. Due to line quantum sensors comprising a square grid, two of the four sensors rested on the opposing orthogonal two at the distal portions of the sensors. To correct the data for self imposed line sensor shading, the exact configuration was placed in an open field on the PVC telescopic rack during solar noon to derive a shading correction 


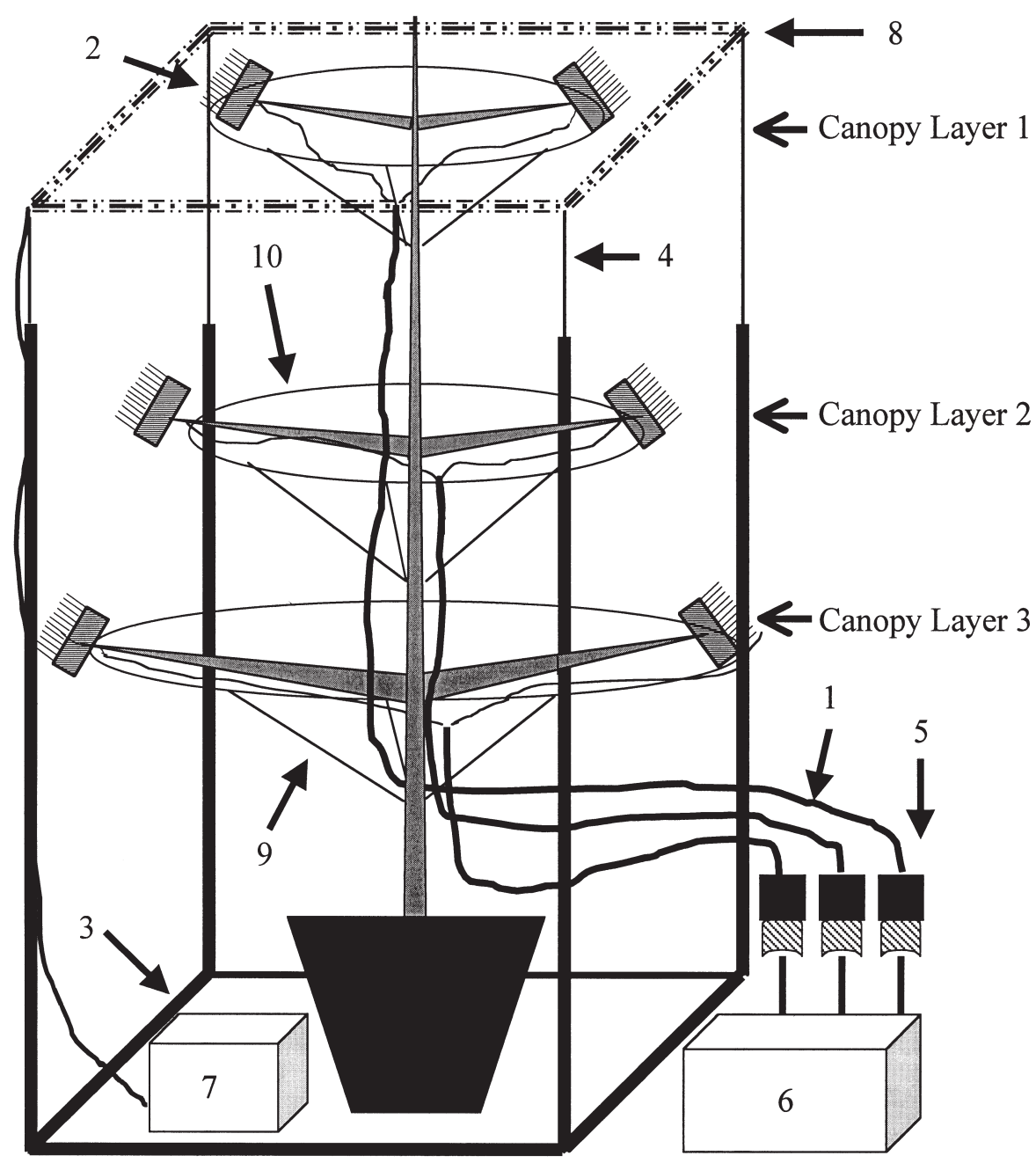

Fig. 1. Diagram of the fiberoptic-based system and line sensor sampling surrounding a study tree. The fiberoptic-based system was located at the median of three canopy layers, the PAR sensors with their associated datalogger were located at the base of the study trees. Fiberoptics were placed on the $\mathrm{N}$, $\mathrm{S}, \mathrm{E}$, and $\mathrm{W}$ direction and the scale diagram only depicts two directions for picture clarity purposes, i.e., there were for fanned microfiber sets per canopy layer. Arrows with referenced numbers are as follows: 1) Corning fiberoptic cable, 2) fanned microfibers, 3) base of PVC support rack, 4) telescopic PVC height extensions, 5) quantum sensor/optical fiber assembly, 6) CR7x datalogger, 7) CR10x datalogger, 8) orthogonal grid of line quantum sensors, 9) wooden dowel, and 10) circular microfiber tree/dowel support system.

factor and data were adjusted accordingly. The potential for light loss due to fiberoptic alignment with the quantum sensor window was corrected for by developing a scaling factor for each individual quantum sensor per fiber bundle (nine total). The system was placed in an open field during solar noon and fiber tips were pointed towards the sun on a clear sunny day. Data were first sampled through the fiberoptics every $1 \mathrm{~s}$ for $1 \mathrm{~min}$ and averaged. The quantum sensor was then removed from the fiber bundle and again sampled for the next minute as above. Data were adjusted for light loss for each of the nine sensors.

\section{Results}

The relationship between line quantum sensor PAR values and those integrated by the fiberoptics presented linear relationships for each layer (Fig. 2). The range in $r^{2}$ values was 0.86 to 0.98 for the three canopy positions and three trees under study. It should be noted that the measurement techniques were not identical of the comparison demonstrates that the fiberoptic system did function and was capable of integrating PAR on a canopy layer basis. It should be noted that a direct 1:1 comparison was not expected as we paired the techniques for independent measurement purposes.

The economics of the fiberoptic system was far cheaper than those of line quantum sensors. The fiberoptic material is sold by length at about $\$ 16 / \mathrm{m}$ which is a fraction of the cost of a line sensor, making the datalogger and PAR sensors the main expense of the fiberoptic system. For example, only one quantum sensor and one fiberoptic bundle are required to intercept the light at a canopy layer, whereas four line sensors were needed to make the same measurements (Baldocchi and Collineau, 1994). In our study, we used $27 \mathrm{~m}$ to instrument three trees and three canopy layers ( $\$ 432$ in fiberoptics and $\$ 900$ in quantum sensors for a total cost of $\$ 1,332$ ). The same cost to outfit all layers with an orthogonal grid of line sensors would be about $\$ 49,500$. Thus, the difference in material cost for our experiment was $97 \%$ less for the fiberoptic bundle and quantum sensor arrangement as compared to orthogonal grids of line sensors. In addition, we instrumented three layers on three individual trees in $5 \mathrm{~h}$. After initial installation, only periodic maintenance of dowel attachments was required and this was mainly associated with storm events. No labor was required to move the fiberoptics to a new layer like that of the orthogonal grid of line sensors and no moving parts exist limiting energy requirements to that of the data logging system.

Adequately characterizing the light environment under or within a canopy requires photosynthetic photon flux density to be sampled in both space and time for several reasons. First, the light penetration through canopies and the effects of sunflecks are sometimes subject to penumbral effects (Baldocchi and Collineau, 1994). These effects are exacerbated in tall fine-leaved canopies. Fiberoptic-based systems, such as the one presented herein, improves PAR sampling ability in canopy gaps. Second, validations of models, such as the Norman and Welles (1983) three-dimensional radiation transfer model justify the need for an inexpensive and integrated PAR measurement technique. Lastly, the inexpensive nature of fiberoptics may allow many more light interception points to be sampled and over time and yield a better representation of the light environment within or among plant canopies. In conclusion, our study indicates that an integrating fiberoptic method may allow complex plots to be better sampled and we envision that an entire nursery, orchard, or forest could be adequately sampled using this technique.

\section{Literature Cited} tion (Bauerle et al., 2003) when line sensor values were saturating.

\section{Discussion}

Our study used orthogonal line sensors to compare an average line sensor value at a specific canopy layer to that of the fiberoptic-based PAR measurement. The linear 1:1 relationship 


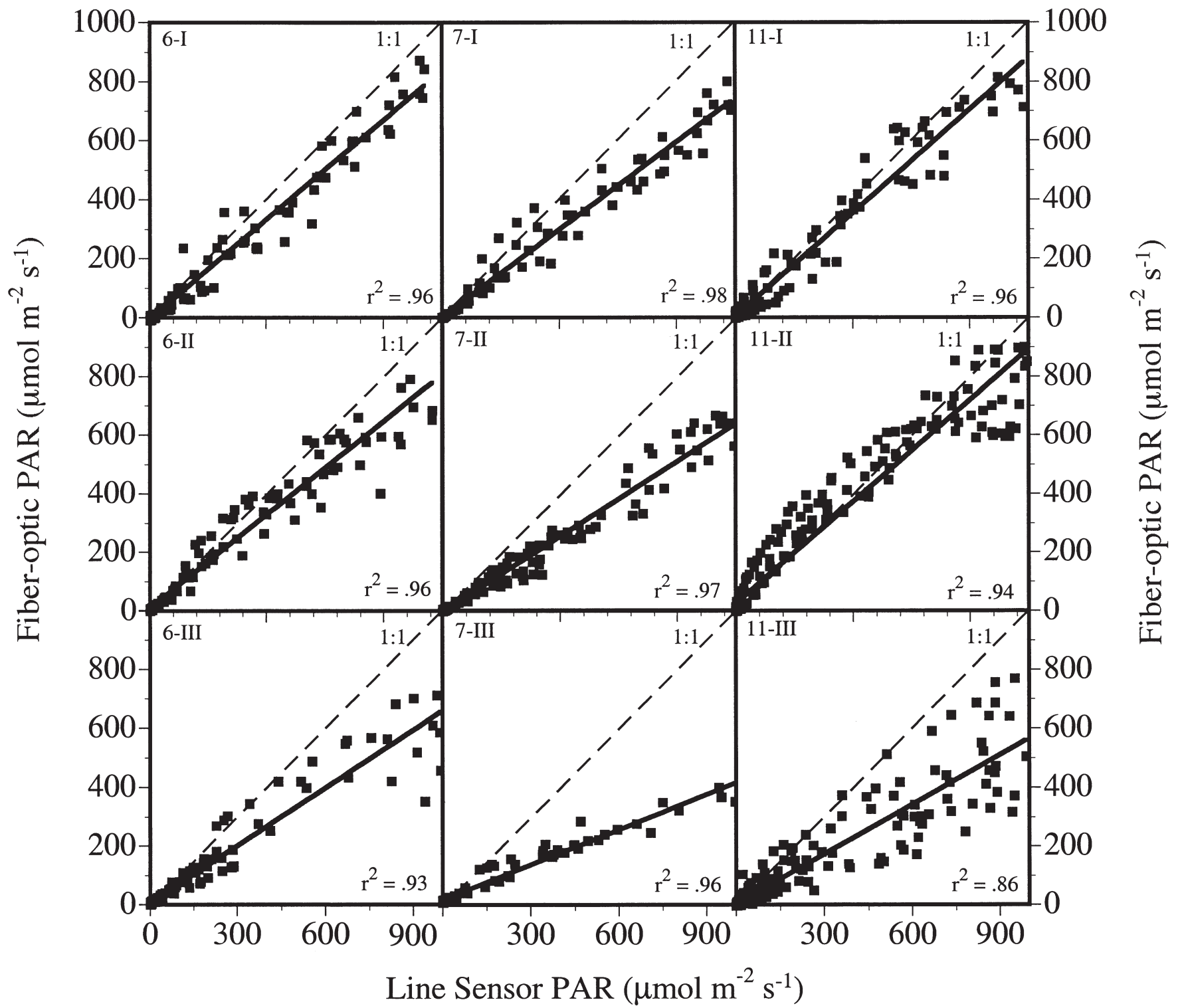

Fig. 2. Linear regression (solid black line) of the relationship between the photosynthetically active radiation value recorded by the fiberoptic-based integration system on the y-axis versus the average of an orthogonal arranged grid of line quantum sensors at three canopy layers (I, II, and III) and for three plot trees $(6,7$, and 11$)$ on the $\mathrm{x}$-axis. The broken line represents the $1: 1$ relationship for each figure.

J.E. Toler. 2004. Modeling intracrown and intracanopy interactions in red maple: Assessment of light transfer on carbon dioxide and water vapor exchange. Tree Physiol. 24:589-597.

Bauerle, W.L., C.J. Post, M.F. McLeod, J.B. Dudley, and J.E. Toler. 2002. Measurement and modeling of the transpiration of a temperate red maple container nursery. Agr. For. Meteorol. 114:45-57.

Bauerle, W.L., T. H. Whitlow, T.L. Setter, T.L. Bauerle, and F.M. Vermeylen. 2003. Ecophysiology of Acer rubrum L. seedlings from contrasting hydrologic habitats: Growth, gas exchange, tissue water relations, abscisic acid, and carbon isotope discrimination. Tree Physiol. 23:841-850.

Corning Cable Systems. 2001. Corning cable systems. SRP 005-006, Hickory, N.C.

Gutschick, V.P., H.P. Barron, D.A. Waechter, and
M.A. Wolf. 1985. Portable monitor for solar radiation that accumulates irradiance histograms for 32 leaf-mounted sensors. Agr. For. Meteorol. 33:281-290.

Jackson, J.E. 1980. Light interception and utilization by orchard systems. Hort. Rev. 2:209-267.

Kyle, W.J., J.A. Davies, and M. Nunez. 1977. Global radiation within corn. Boundary Layer Meteorol. 12:25-35.

Norman, J.M. and J.M. Welles. 1983. Radiative transfer in array of canopies. Agron. J. 75:481-488.

Pearcy, R.W. 1989. Radiation and light measurements, p. 97-116. In: R.W. Pearcy, J. Ehleringer, H.A. Mooney, and P.W. Rundel (eds.). Plant physiological ecology: Field methods and instrumentation. Chapman \& Hall, New York. 